\title{
Report on the 13th World Congress in Fetal Medicine, Nice, 2014
}

\author{
Bijoy K. Balakrishnan
}

Received: 8 September 2014 / Accepted: 29 September 2014/Published online: 26 November 2014

(C) Society of Fetal Medicine 2014

The world of fetal medicine is continuously evolving with frequent introduction of newer concepts and techniques. It is not possible for busy practitioners to keep themselves abreast with current developments and standards of practice on a day-to-day basis. Hence, conferences like the World Congress in Fetal Medicine organized by the Fetal Medicine Foundation annually are a very valuable educational exercise that should not be missed by true proponents of fetal medicine. In this article, some topics discussed at the Congress are covered:

1. Monochorionic twins (MC twins)

2. Fetal diagnosis and therapy

3. Growth restriction

4. Neurosonogram

5. Noninvasive prenatal testing (NIPT)

\section{Monochorionic Twins}

(Speakers: Liesbeth Lewi, Cristina Rossi, Edward Gratacos, and Kypros Nicolaides)

A number of complications are unique to $\mathrm{MC}$ twins like discordant anomaly, twin-twin transfusion syndrome (TTTs), twin anemia polycythemia sequence (TAPS) and selective intrauterine growth restriction (sIUGR). An even more confusing problem is when two or more complications coexist. For instance, if TAPS and TTTs occur in the

B. K. Balakrishnan $(\square)$

Department of Feto-Maternal Medicine, CIMAR Fertility

Centre, Off NH 17, Behind Thykkavu Bus Stop, Cheranellore

PO, via Edappally, Kochi 682034, Kerala, India

e-mail: drbijoykb@rediffmail.com same patient which complication would you treat first? This dilemma is put to rest by the classification presented below.

Discordant anomaly $\rightarrow$ TTS $\rightarrow$ TAPS $\rightarrow$ sIUGR

The sequence of complications mentioned above serves as a guide when confronted with two or more conditions coexisting in the same pregnancy. As mentioned in the previous example when both TTTs and TAPS occur together, as per this list one would treat TTTs.

\section{Discordant Anomaly}

The most common anomaly in monochorionic twins is the cardiac anomaly and hence a detailed fetal echo is a must in these pregnancies. The management of anomalies that are picked up early is selective fetal reduction. Unlike in dichorionic twins (DC twins), in $\mathrm{MC}$ twins $\mathrm{KCl}$ cannot be used. One must attempt to do reduction using bipolar cautery or high frequency radio ablation (HFRA) or Laser. There is a risk of fetal loss of the normal baby, which is around $10-20 \%$. However, the risk for preterm birth is the highest for cautery.

\section{TAPS}

With an incidence of around $5 \%$, TAPS is a complication that usually occurs after 26 weeks. The diagnosis is based on the ultrasound findings outlined in Table 1. A practical advice in diagnosing TAPS is to revaluate the patient again after 3-4 days before confirming the diagnosis, as there is a high chance of false positivity.

Management options are to either go in for Laser to burn out the small AV anastomosis causing TAPS or to go in for intrauterine fetal blood transfusion. It is important to 
Table 1 Ultrasound findings in TAPS

\begin{tabular}{ll}
\hline Anemia & Polycythemia \\
\hline MCA $>1.5$ MOMs & $<1$ MOMs \\
Placenta Thick echogenic & Hypoechogenic \\
Liver Enlarged & Star sky pattern \\
\hline
\end{tabular}

remember that both the above mentioned treatment options are used only before 30 weeks; after 30 weeks delivery is a better option. Laser is a difficult option in this case as the $\mathrm{AV}$ anastomosis is quite small and may not be easily identified on fetoscopy. Hence, transfusion is the treatment of choice but if one has to perform more than two transfusions and the fetus is still less than 30 weeks, Laser may be attempted as a second line of management. It must also be emphasized that the risk for polycythemia and autoamputation in the recipient is present but not that common as to prevent transfusion.

\section{sIUGR}

sIUGR is classified into three types based on their Doppler abnormalities.

Type I Growth discordance with normal Doppler

Type II With persistent AEDF/REDF

Type III With intermittent AEDF

In type I sIUGR, the management of choice is serial surveillance and timely delivery. In type II and III there is an option to go for conservative expectant management as outlined above or to go in for active management like Laser or cord occlusion. Type II and III are further subclassified based on the following criteria:

(i) Gestational age less than 24 weeks; (ii) Discordance $>35 \%$ and (iii) REDF/DV PI $>95$ th centile

If any one of the above-mentioned complications are present in type II and III then one must opt for laser or cord occlusion. Cord occlusion is preferred over Laser because even after Laser the smaller twin may die as it has a smaller placental mass.

\section{Fetal Diagnosis and Therapy}

In this section, the recent developments in the diagnosis and management of common anomalies are discussed.

\section{Esophageal Atresia (EA)}

(Speakers: Emmanuel Spaggiari, Daryl Armstrong Scott) There are five types of EA as outlined in Fig. 1. Diagnosis of EA is based on a combination of USG findings like small or absent stomach with hydraminos and the presence of the pouch sign. However these signs are not always present especially in the type III variant, which is the most common type of EA. It was proposed to assess the amniotic fluid for biomarkers like gamma glutamyl transpeptidase (GGTP) and alpha fetoprotein (AFP). The EA index is the combination of these markers.

EA index: GGTP $\times$ AFP

If the index is greater than 3 then the possibility of EA is very high.

\section{Gallbladder (GB)}

(Speakers: Francoise Muller, Eran Ashwel)

The Gallbladder is routinely seen on USG but rarely documented but two papers were presented at the Congress, which highlighted the need to document the presence or absence of the GB. The GB may be physiologically absent but this occurs only $0.5 \%$ of the time and a repeat scan after some time would enable its demonstration. If the GB is not visualized then one must think of the following possibilities:

(i) GB agenesis; (ii) Cystic fibrosis; (iii) Biliary atresia (iv) Aneuploidies (triploidies and 47XYY)

Hence the work up of these fetuses would start with a basic karyotype followed by an $\mathrm{AF}$ assessment of the biomarkers GGTP and aminopeptidase $\mathrm{M}$ if the patient presents before 22 weeks. In biliary atresia these biomarkers will be absent. But after 22 weeks these markers would be absent even in normal fetuses as a mature anal sphincter will prevent the leakage of these markers into the amniotic fluid. Therefore after 22 weeks a fetal blood estimation of GGTP may be required.
Fig. 1 Types of esophageal atresia
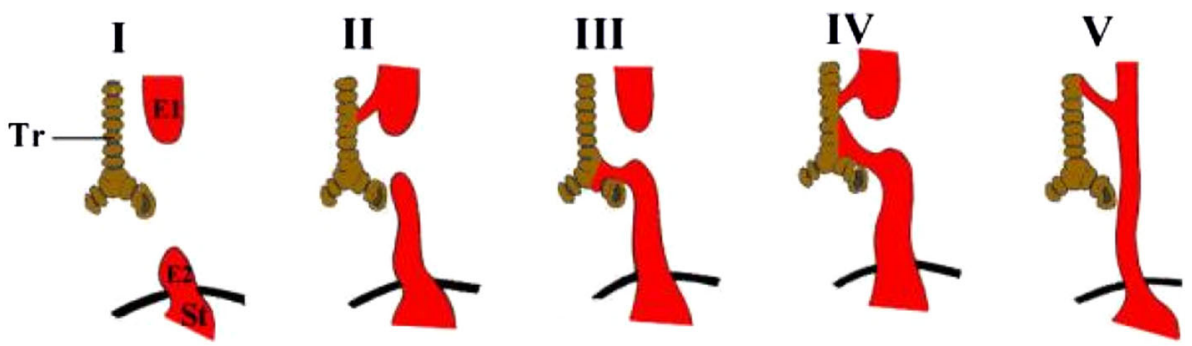

Tr - trachea E1 - proximal esophagus E2 - distal esophagus St - stomach 


\section{Sacrococcygeal Teratoma (SCT)}

(Speakers: Francois Luks, Greg Ryan)

There are four types of SCT based on the external and internal component of the tumor (Fig. 2). Type I and II are more common antenatally. The complications specific to SCT are hydrops and mirror syndrome secondary to the vascular steal effect.

The prognosis of SCT depends largely on the size of the tumor and its rate of growth. Large tumors greater than $7 \mathrm{~cm}$ with increased vascularity have a poorer prognosis. The rate of growth is assessed by calculating the volume of the tumor using the formula $L \times W \times H \times 0.52 \mathrm{~cm}^{3}$. If the rate of growth is greater than $150 \mathrm{~cm}^{3} /$ week then the mortality rate is around $60 \%$. In these cases the treatment options are to either go for open fetal surgery or minimally invasive therapy like HFRA/laser or insertion of thrombogenic coils to the feeding vessels.

\section{Lung Lesions}

(Speakers: Annegret Geipel, Francois Luks)

The most common lung lesions encountered are congenital pulmonary adenamatoid malformation (CPAM) and bronchopulmonary sequestration (BPS). An important point to note in these lung lesions is that they do not cause pulmonary hypoplasia unlike other masses in the thorax viz. $\mathrm{CDH}$.

Congenital pulmonary adenomatoid malformation is of three types macrocystic type $(50 \%)$ microcystic type (25\%) and the mixed type (25\%). Most of the time $(85-90 \%)$, one lobe is affected which is usually the lower lobe. Complete resolution occurs in CPAM $50 \%$ of the time especially in the microcystic type. The survival rate in CPAM is good at around $90 \%$. Of the fetuses with CPAM, $5-10 \%$ do develop hydrops especially the macrocystic
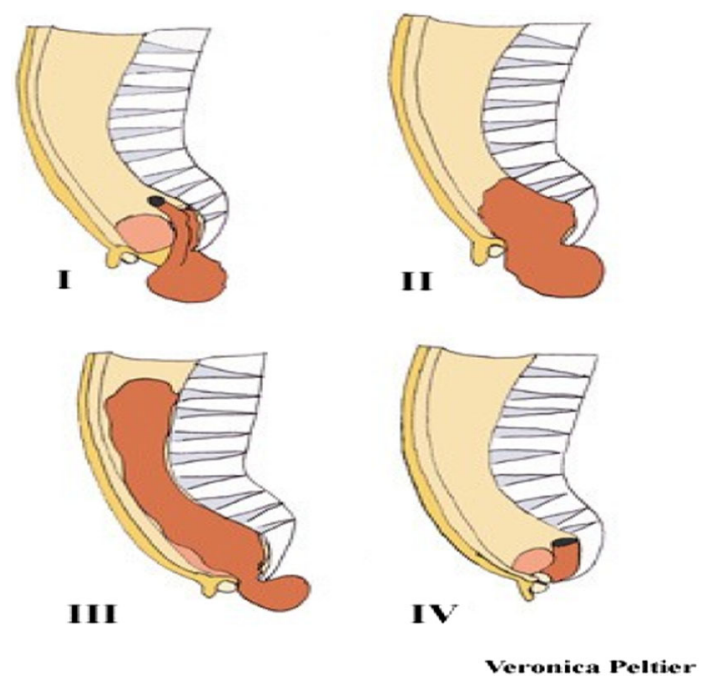

Fig. 2 Types of sacrococcygeal teratoma

variant. Medical management is available in the microcystic type where treatment with maternal steroid injection has shown to cause resolution within 30 days even in hydropic fetuses. BPS is usually a benign hyperechoic lesion present on mostly the left side and regresses $40-50 \%$ of the time. Sometimes they do cause pleural effusion in which case thoracoamniotic shunting may be done.

\section{Urinary Tract Defects}

\section{(Speaker: Christoph Berg)}

Hydronephrosis is a common problem that leads to a lot of confusion on management and hence the segregation into mild, moderate and severe will help in counseling the patients (Table 2). However, the prognosis is not based just on USG findings or AF assessment and a fetal urine evaluation would be needed to predict renal function. The most specific fetal urine marker is beta-2-microglobulin.

\section{USG at 8-9 Weeks}

(Speaker: Bernard Benoit)

An early USG at 8-9 weeks is very helpful in predicting the prognosis. The following USG markers were suggested:

(i) Irregular or slow heart rate; (ii) Irregular head; (iii) Abnormal brain cavities; (iv) Early hydrothorax; (v) Small gestational sac compared to embryo; (vi) Small embryo compared to gestational age

\section{Fetal Reduction}

\section{(Speakers: Mark Evans, Luc De Catte)}

There is a lot of controversy in fetal reduction for triplets with conflicting opinions on whether to reduce triplet pregnancies or not. This issue was put to rest by a large retrospective trial that analyzed over 1,000 fetal reductions done by 26 operators. The main conclusion of this trial was that reduction in triplets to twins did not improve the survival when compared to nonreduced triplets but when you look at the morbidity of reduced versus nonreduced triplets the morbidity was significantly increased in the nonreduced triplets due to a high incidence of prematurity in this group.

Another area of concern in triplets is the management of a triplet pregnancy with a monochorionic pair. The options available are:

Table 2 Classification of hydronephrosis

\begin{tabular}{lll}
\hline Hydronephrosis & 2nd trimester $(\mathrm{mm})$ & 3rd trimester $(\mathrm{mm})$ \\
\hline Mild & $4-7$ & $7-9$ \\
Moderate & $7-10$ & $9-15$ \\
Severe & $>10$ & $>15$ \\
\hline
\end{tabular}


Continue pregnancy (Risk of triplets + MC complications) Reduce singletons (Risk of reduction + MC complications) Reduce the MC pair (Risk of reduction)

Of all the options available reduction of the MC pair has the least complication, however the wishes of the parents will also have to be considered in this scenario.

\section{Growth Restriction}

(Speaker: Edward Gratacos)

A protocol-based management was suggested for early onset IUGR. Fetuses with early onset IUGR are classified into four stages based on their Doppler findings. The stage decides the frequency of surveillance and even the timing and mode of delivery as illustrated in Fig. 3 and Table 3.

\section{Neurosonogram}

(Speaker: Rabih Chaoui)

A detailed review of the fetal CNS was done at the congress with special emphasis on three areas, the CSP (cavum septum pellucidum), sylvian fissure and the posterior fossa.

\section{CSP}

The CSP is linked to the prosencephalon development and the absence of CSP is a pointer to abnormal cleavage of the

Fig. 3 Stages of IUGR

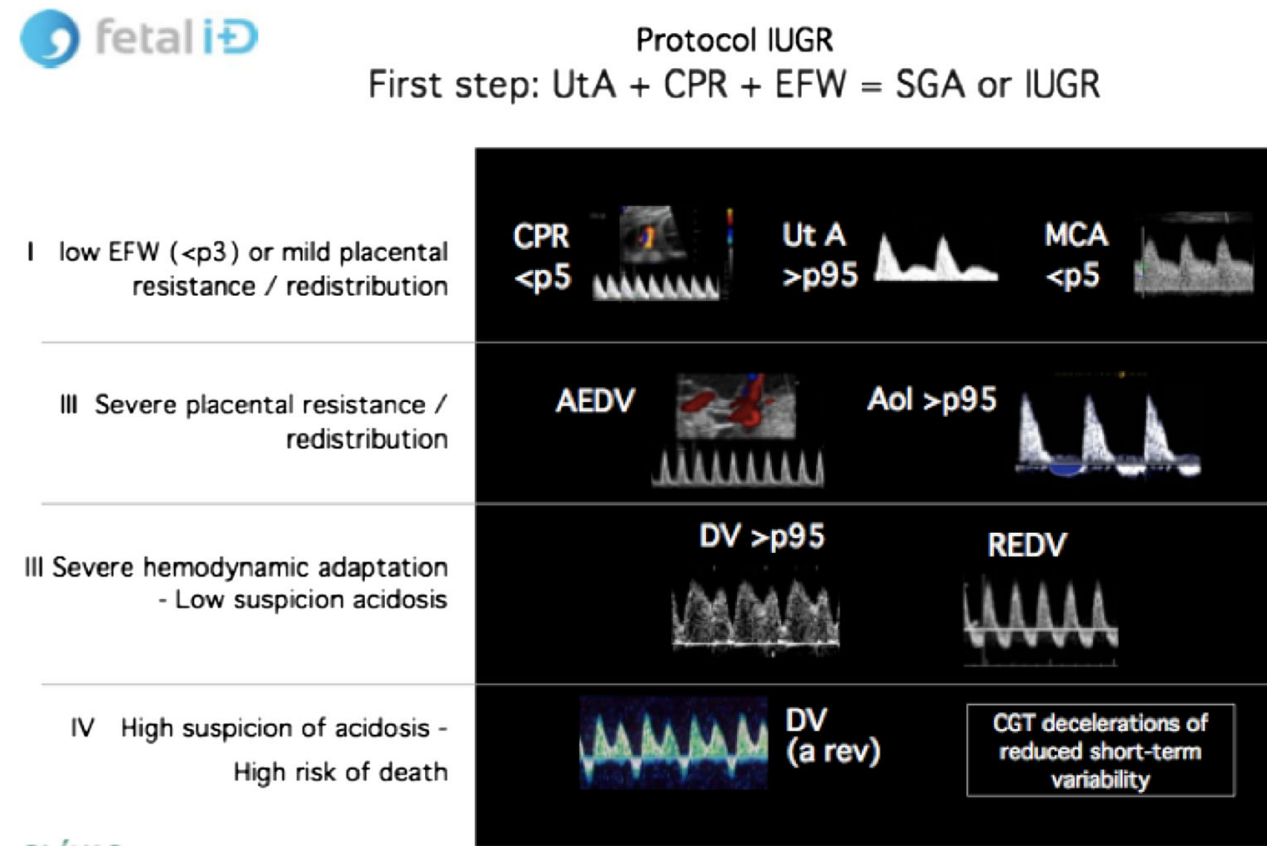

Fig. 4 Flow chart for diagnosis of agenesis of septum pellucidum. ACC Agenesis of the corpus callosum, $P M G$ Polymicrogyria, SOD Septooptic dysplasia, SP Septum pellucidum

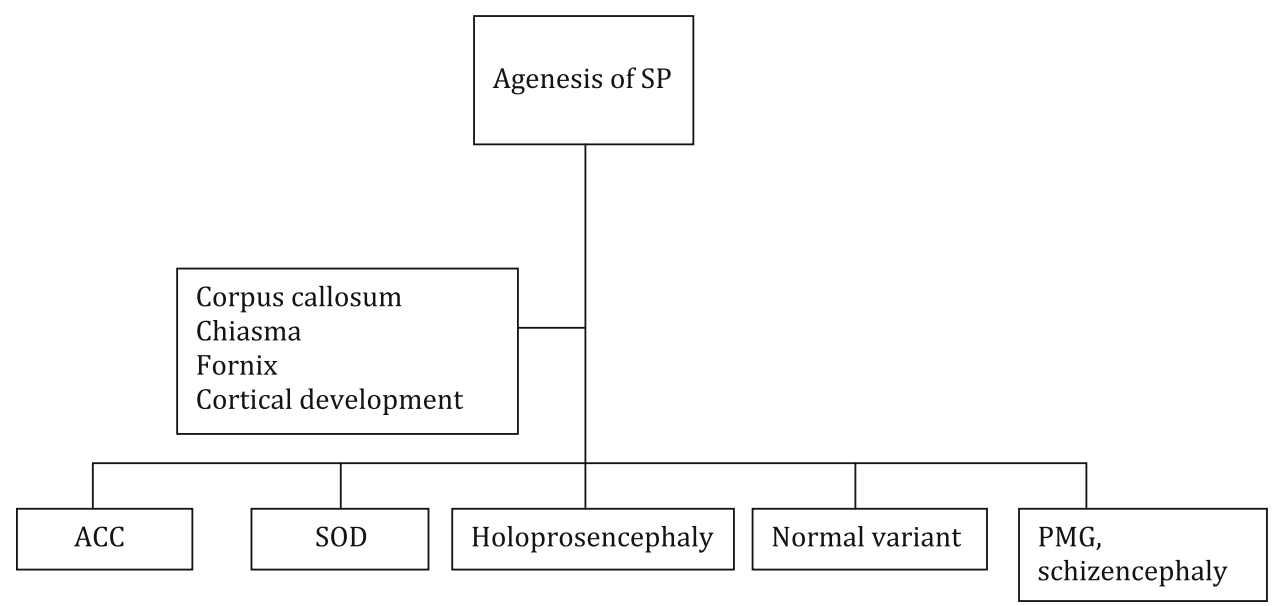


Table 3 Management protocol for IUGR according to severity stages

Fig. 5 The Sylvian fissure is assessed in the plane seen above where the ambient cistern (AC), third ventricle (TV) and the columns of fornix $(\mathrm{F})$ are seen

\section{IUGR}

\section{Management protocol according to severity stages}

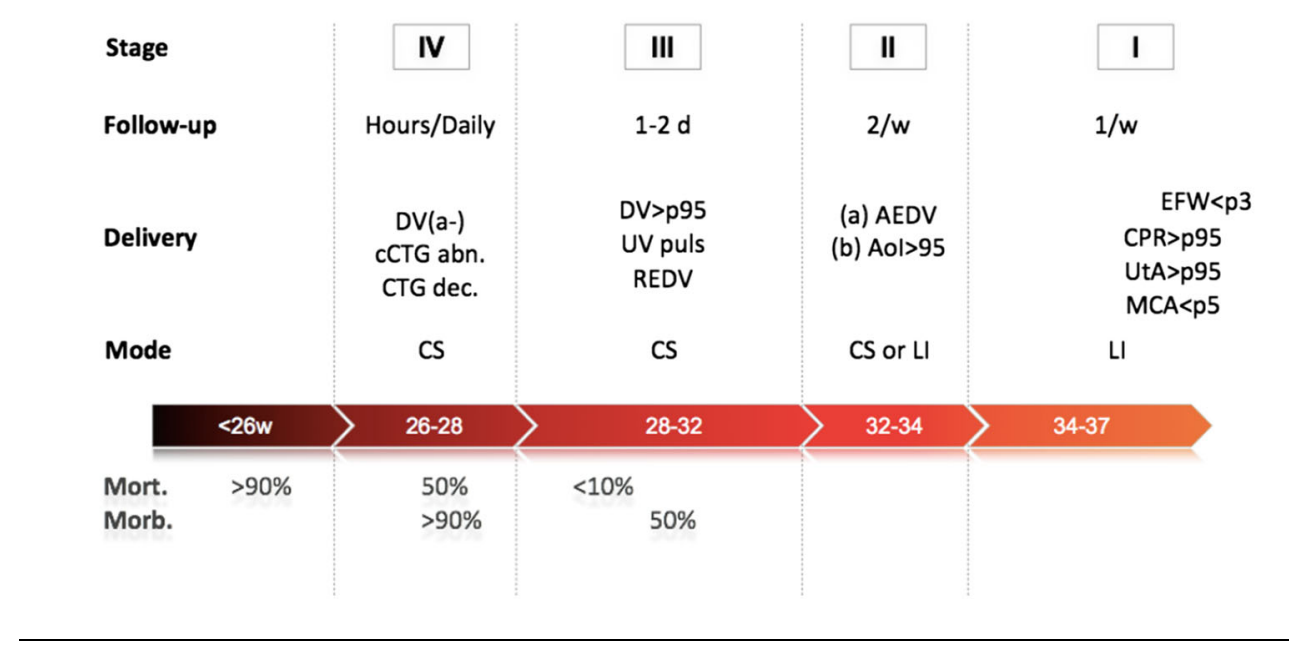

forebrain, separation of the ventricles, development of the corpus callosum, optic chiasma and cortical development. The flow chart (Fig. 4) gives one an idea on what to look for when the diagnosis of agenesis of septum pellucidi is made.

Isolated agenesis of CSP has a good outcome hence it is important to rule out more serious conditions like septooptic dysplasia where the optic chiasma is absent or holoprosencephaly where the fornix are absent with agenesis of corpus callosum or migration disorders like schizencephaly or polymicrogyria.

\section{Sylvian Fissure}

Migration disorders are late onset CNS anomalies that can be missed on routine mid-trimester targeted scanning. 
Hence, it important to look for markers that help us identify these abnormalities. One such marker is the Sylvian fissure. The exact plane in which this fissure should be evaluated was explained. The ambient cistern, the third ventricle and the fornix should be visible while assessing the Sylvian fissure as illustrated in Fig. 5.

\section{Noninvasive Prenatal Testing (NIPT)}

(Speakers: Edward Gratacos, Kypros Nicolaides)

Noninvasive prenatal testing is now widely available and there is actually a lack of consensus on how to apply this new technology. It is important to understand a few important points regarding NIPT. NIPT is a screening test, which identifies fetuses that are at high risk for trisomy 21 but even when the test is positive a confirmatory amniocentesis must be done. The sensitivity for trisomy 13 and sex chromosomes is less than that of Down syndrome. NIPT should not replace the 11-14 weeks scan as this scan screens not only for aneuploidies but also for a host of structural abnormalities, fetal and maternal complications.
It was suggested at the Congress that NIPT can be used in a contingent model as described below whereby the cost of using NIPT on the whole population can be brought down while at the same time, the number of invasive procedures are not significantly increased.

Contingent Screening using NIPT:

Step 1 Do a combined test at 11-14 weeks

Step 2 Assign a cut off of 1:10 for invasive procedures

Step 3 Patients with values between 1:10 and 1:1,000 have NIPT

Step 4 Patients with values greater than 1:1,000 have no further testing

By using this contingent model NIPT needs to be used only for $25 \%$ of the population with a detection rate for Down syndrome of around $90 \%$ and an invasive testing rate of $0.8 \%$.

Conflict of interest None 